\title{
EDITORIAL
}

\section{Preface for the special issue on regenerative medical materials}

(C) Higher Education Press and Springer-Verlag Berlin Heidelberg 2014

B iomaterials science is undoubtedly at the frontier of materials science and engineering. It bridges materials science with biology and medicine, and more importantly its progress brings direct impact to improving health and quality of life for many of us. Traditionally, biomedical implants are made from common engineering materials to replace human tissues or organs. A good example is the artificial joints made of cobalt-chromium alloy, titanium alloy, and high density polyethylene. Over 1 million total hip arthroplasties are performed annually in North America. They effectively relieve pain and restore mobility of those suffering from osteoarthritis and osteoporosis. Other well-known biomedical implants developed in the 20th century are dental implants, artificial heart valves, coronary stents, and artificial hearts. Although effective in achieving the functions, these biomaterials share the same drawback: they are inert and do not actively interact with the surrounding tissues. The injured tissues or organs are replaced permanently by non-viable materials without the possibility of regeneration.

A new development in the past two decades has been the concept of regenerative medicine. Driven by the idea of living healthy and natural, and built on the latest progress in biology and medicine especially stem cell research, regenerative medicine aims at regenerating functional tissues and organs. According to the National Institutes of Health, regenerative medicine is the process of creating living, functional tissues to repair or replace tissue or organ function lost due to age, disease, damage, or congenital defects. At the core of such a process is the tissue engineering strategy. There are three key components that need to be well-orchestrated to achieve the goal: human cells to be infused to achieve the specific functions, biomaterials scaffolds to support and mediate cell functions, and growth factors to facilitate cell differentiation. Although centered on human cells, the success of tissue regeneration also heavily relies on active participation of biomaterials scaffolds in the process. The scaffold with best performance has been the decellularized matrices. How can we design and make biomaterials with similar functions as extracellular matrices? Rapid progress in regenerative medicine poses challenges and opportunities to the biomaterials community.

In the past ten years, we have seen increased interest in tissue engineering and regenerative medicine from the biomaterials community. Such a shift in focus is shaping the future of biomaterials research and development. We feel that biomaterials for regenerative medicine, or regenerative biomedical materials, are at the frontier of biomaterials research. It is therefore the purpose of this special issue to present you with the latest progress of this field. The topics 
included in this special issue cover various aspects related to regenerative biomedical materials, from nano-materials, conductive polymers, responsive scaffolds, to growth factors delivery, stem cell culture. The applications also range from nerve regeneration, cardiovascular systems, to orthopaedics and dentistry. Hopefully, this issue on regenerative medical materials of the international journal Frontiers of Materials Science will serve to stimulate active discussions in the materials community and to inspire young researchers to join this dynamic and exciting field.

Shengmin ZHANG, Huazhong University of Science and Technology, China and

Rizhi WANG, The University of British Columbia, Canada March, 2014 